\title{
Electromagnetic field redistribution in hybridized plasmonic particle-film system
}

\author{
Yurui Fang ${ }^{1,2}$ and Yingzhou Huang ${ }^{1, a)}$ \\ ${ }^{1}$ Department of Applied Physics, College of Physics, Chongqing University, Chongqing 400044, China \\ ${ }^{2}$ Division of Bionanophotonics, Department of Applied Physics, Chalmers University of Technology, \\ Gothenburg SE-412 96, Sweden
}

(Received 8 January 2013; accepted 5 April 2013; published online 19 April 2013)

\begin{abstract}
Combining simulation and experiment, we demonstrate that a metal nanoparticle dimer on a gold film substrate can confine more energy in the particle/film gap because of the hybridization of the dimer resonant lever and the continuous state of the film. The hybridization may even make the electric field enhancement in the dimer/film gap stronger than in the gap between particles. The resonant peak can be tuned by varying the size of the particles and the film thickness. This electromagnetic field redistribution has tremendous applications in sensor, photocatalysis and solar cell, etc., especially considering ultrasensitive detection of tracing molecule on substrates. (C) 2013 AIP Publishing LLC [http://dx.doi.org/10.1063/1.4802267]
\end{abstract}

Thanks to modern material synthesis techniques, the fast development of modern nanofabrication technologies and high precision characterization techniques, plasmonics has during the last ten years become an innovative, vigorous, crossdisciplinary scientific field, involving physics, chemistry, biology, etc. ${ }^{1-5}$ The huge electric field enhancement which is caused by surface plasmons has numerous applications in various research fields, such as single molecule detection, ${ }^{6,7}$ photocatalysis, ${ }^{8}$ photovoltaics, ${ }^{9}$ surface enhanced spectroscopies, ${ }^{10}$ enhanced transmission, ${ }^{11}$ sensing ${ }^{12}$ optical trapping, ${ }^{13}$ etc.

Since the plasmonic properties are highly related to the metal nanostructures' composition, geometry, and dielectric function, ${ }^{14}$ the field enhancement has been studied in different plasmonic systems, such as individual particles, ${ }^{15}$ dimers, ${ }^{16-20}$ trimers, ${ }^{21}$ and films. ${ }^{22}$ Because of the good application prospect, the particle-film system has aroused the interest of researchers from all over the world. ${ }^{23-25}$ Even though a lot of particle-film system configurations have been studied, the enhancement in those works was mainly attributed to the coupling between particles, particle tips or between the particle and the film using an out-of-plane polarization. ${ }^{26-29}$ However, the hybridization between particle interactions and the film with an in-plane polarization is seldom considered. In this paper, the redistribution of electromagnetic (EM) energy in a metal particle-film system is studied using a normal incidence illumination configuration. The difference between the EM field distribution in a single particle-film system and that in a dimer particle-film system is described. The redistribution of EM field is explained by simulated energy density and surface charge distributions combining with experimental results of Surface Enhanced Raman Spectroscopy (SERS) measurements. The hybridization of the particle dimer and the film causes redistributions of the local electric (E) field, which in turn creates stronger field enhancement factors in the particle-film gap than in the gap between the particles. The hybridization is investigated

\footnotetext{
${ }^{\text {a) }}$ Author to whom correspondence should be addressed. Electronic mail: yzhuang@cqu.edu.cn
}

by varying the diameter of the particles and the thickness of the film. The system investigated in this paper gives a basic idea and indication of field enhancements in particle-film systems.

All systems considered in this work were simulated using the finite element method (COMSOL 4.3 A commercial package). The studied geometry is comprised of a gold film on top of a glass substrate, with one or two silver spheres located $1 \mathrm{~nm}$ above the film, with a $1 \mathrm{~nm}$ edge-to-edge dimer separation. The illumination was incident from the particle side, normal to the substrate, with a polarization along the dimer axis and an electric component $1 \mathrm{~V} / \mathrm{m}$. Figure 1(a) shows the electric field distribution of an $\mathrm{R}=30 \mathrm{~nm}$ silver sphere on a $30 \mathrm{~nm}$ thick gold film. The incident direction and polarization are shown in the figure with red and blue arrows, respectively. The excitation wavelength is $633 \mathrm{~nm}$. The electric field is mostly confined in the gap between the particle and the film. However, since the lobes of the dipole mode, modified by the gold film, are still on the two sides of the silver sphere, parallel to polarization, the electric field in the narrowest position of the gap is not the strongest. If two particles are put above the film with a $1 \mathrm{~nm}$ dimer separation, as seen in Figure 1(b), the electric field between the two particles is much stronger than the single particle's maximum enhancement. Surprisingly, the electric field between the particle and the film is also very strong, even though the polarization is parallel to the film. By studying the electric field in the gaps A, B, and C (Figures 1(a) and 1(b)), we find that not only is the electric field in the gaps of a dimer-film system much stronger than in a single particle-film system (Figure 1(c)) but also, in a broad wavelength range, the $\mathrm{E}$ field in B is even stronger than in C. The maximum enhancement is more than 160 at $680 \mathrm{~nm}$, giving a SERS enhancement factor more than $6.5 \times 10^{8}$. The SERS signals should be doubled in experiment because there are two particle-film gaps in the system. Figure 1(d) shows the ratio of the E field in points $\mathrm{B}$ and $\mathrm{A}$. In most of the condition, the $\mathrm{E}$ field in $\mathrm{B}$ is stronger than in A, with a maximum ratio at $680 \mathrm{~nm}$ of up to 45 times. If the molecules cannot be adsorbed on a 

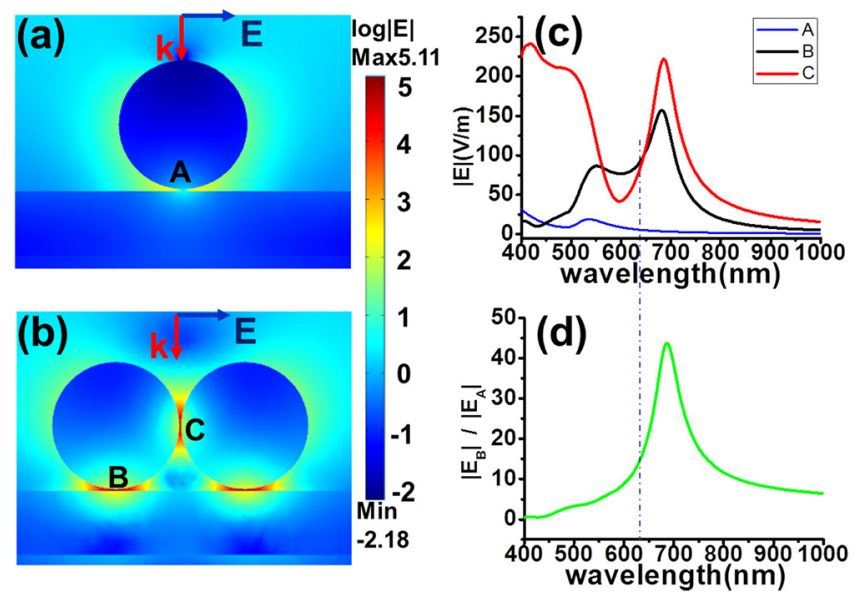

FIG. 1. (a) Electric field distribution of an $\mathrm{R}=30 \mathrm{~nm}$ Ag particle on a $30 \mathrm{~nm}$ thick $\mathrm{Au}$ film with a $1 \mathrm{~nm}$ gap. (b) Electric field distribution of two $\mathrm{R}=30 \mathrm{~nm} \mathrm{Ag}$ particles on a $30 \mathrm{~nm}$ thick Au film with $1 \mathrm{~nm}$ gaps. (c) Electric field enhancement from the gaps indicated in (a) and (b) as A, B, and $\mathrm{C}$. The data in A correspond to the strongest enhancement in (a), slightly deviating from the middle point. (d) The ratio of the electric field in B and A. The vertical dashed line is positioned at $633 \mathrm{~nm}$.

particle, but only on a film, particle dimers can be used to enhance a SERS signal to a much larger extent than only using one particle.

In order to verify the simulated results, experimental SERS measurements were conducted using a similar geometry. Silver nanoparticle colloids were synthesized by citrate reduction of $\mathrm{AgNO}_{3},{ }^{23}$ centrifuged to get the particles, and then diluted with distilled water. For the SERS measurement, first, a glass substrate with a $30 \mathrm{~nm}$ thick gold film was immerged in a $1 \times 10^{-5} \mathrm{M}$ 4-nitrobenzenethiol solution for $2 \mathrm{~h}$ and then rinsed at least 10 times ( 2 min each time) with distilled water in order to avoid thick clusters of molecules on the surface. Then, the particle sol $(\mathrm{R} \sim 30 \mathrm{~nm})$ was dripped and dried on the surface. The Raman spectra from one or two particles were measured with a micro Raman spectrometer with a $633 \mathrm{~nm}$ laser. The results are shown in Figure 2. The red curve is from a dimer-film system and the black curve is from a single particle-film system. If the background is subtracted from the curves, the peak height at $1580 \mathrm{~cm}^{-1}$ enhanced by a dimer, and a single particle is 12000 and 1000, respectively. The integrated peak signal, indicated as a blue area in Figure 2, is 48 times larger in the dimer case, which is smaller than the simulated ratio. The discrepancy may be due to enhancement factor variations depending on shape and surface roughness effects of the

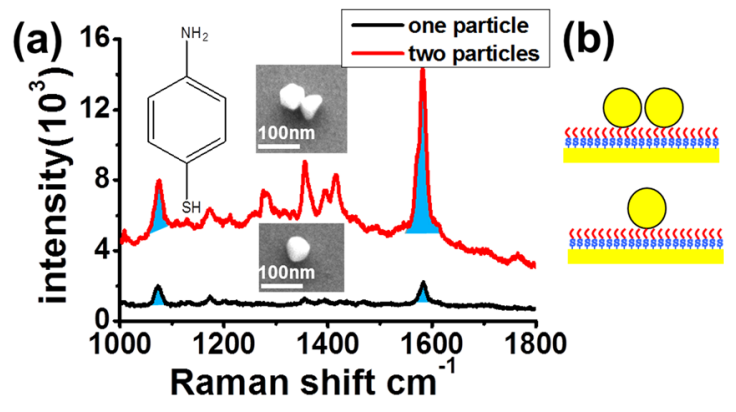

FIG. 2. (a) SERS of 4-nitrobenzenethiol adsorbed on Au film from one or two Ag particles on Au film. (b) Scheme of the samples. particles and the film, respectively. Additionally, the ratio of peaks increases with larger Raman shifts, in agreement with the simulation.

For a deeper understanding on the mechanism of the huge enhancement in the gap between the particle dimer and the film, simulations illustrating surface charge and energy density distributions are plotted in Figure 3. Figure 3(a) shows the surface charge distribution of a single $60 \mathrm{~nm}$ diameter silver particle on a gold film with a $1 \mathrm{~nm}$ gap, which describes the dipole oscillation of the system. Due to the dipole induced in the sphere and the image dipole in the gold film, the effective field is like two coupled dipoles as shown in Figure 3(a) with dashed arrows. Since the gap is not located at the positions of two effective dipoles but in the middle of them, there is no efficient charge induced in the gap, resulting in modest $E$ field strength at this position. In the system comprised of a particle dimer on the film (Figure 3(b)), a much stronger dimer dipole, formed by the two spheres, is coupled to the image dipole in the film. The total effect equals to two effective dipoles coupled together in the direction normal to the film, as the dashed arrows illustrates. Superimposing the effects of gaps and effective dipoles leads to the much stronger enhancement in the dimer-film system. Figure 3(c) shows the electromagnetic energy flows on a particle's surface. It is slightly stronger in the polarization direction. For a silver sphere dimer on the gold film, as shown in Figure 3(d), the two spheres focuses more energy, resulting in more energy confined in the dimer/film gap.

Now, we investigate the mechanism from a hybridization point of view and study the properties of the dimer particle-film system with different sizes of the particles and thickness of the film. Figure 4(a) shows the E field in a gap
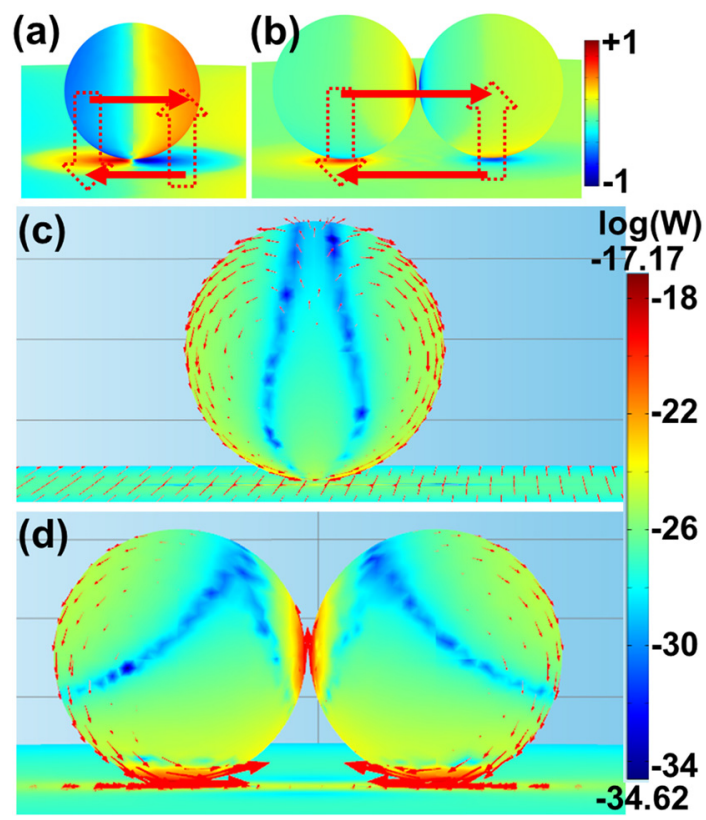

FIG. 3. (a) and (b) Simulated surface charge distributions of one and two Ag spheres $(R=30 \mathrm{~nm})$ on an Au film $(D=30 \mathrm{~nm})$ with $1 \mathrm{~nm}$ gap. Cube root scale is used for a better contrast. The red arrows show the induced dipoles and the dotted arrows show the equivalent dipoles. (c) and (d) Simulated energy density distributions of one and two $\mathrm{Ag}$ spheres $(\mathrm{R}=30 \mathrm{~nm})$ on $\mathrm{Au}$ film $(D=30 \mathrm{~nm})$ with a $1 \mathrm{~nm}$ gap. Logarithmic scale is used for a better contrast. The red arrows show the power flow direction. 

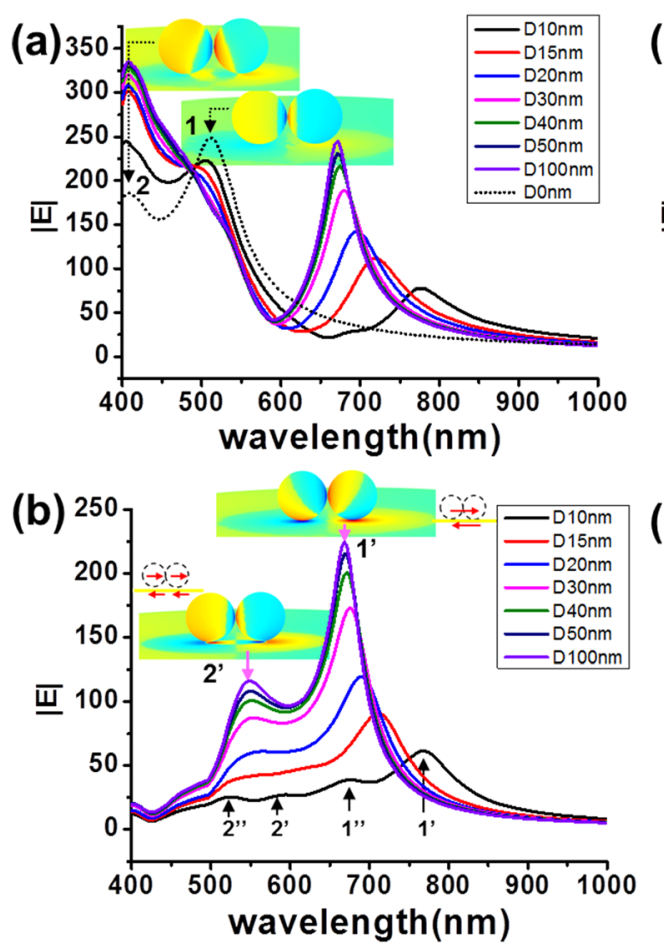

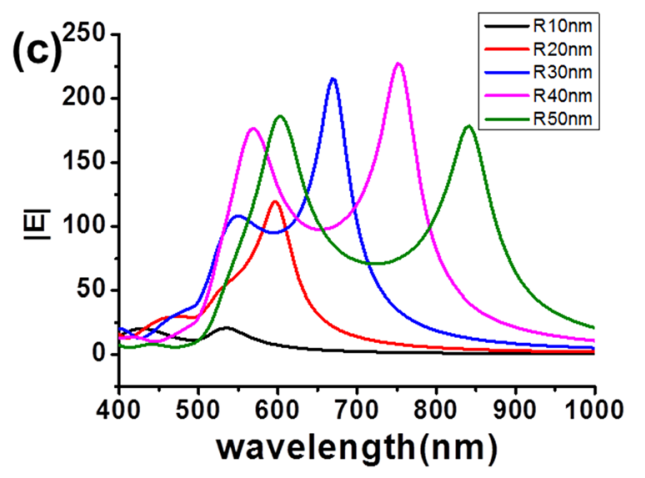

(d)

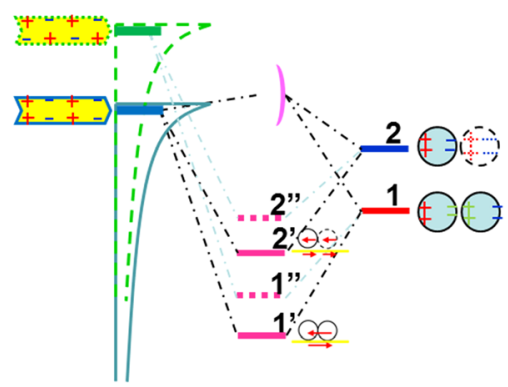

FIG. 4. (a) Simulated electric field from gap $\mathrm{C}$ of $\mathrm{Ag}$ sphere $(\mathrm{R}=30 \mathrm{~nm})$ dimers on different thickness of $\mathrm{Au}$ film. The insets are the surface charge distribution of silver particles on glass substrate at the peak wavelength indicated by the black arrows to show the original resonance without the gold film. (b) Simulated electric field from gap B of Ag sphere $(\mathrm{R}=30 \mathrm{~nm})$ dimers on different thickness of $\mathrm{Au}$ film. The insets are the surface charge distribution $(\mathrm{D}=30 \mathrm{~nm})$ at the peak wavelength indicated by the pink arrows. (c) Simulated electric field from gap B of different sizes of sphere dimers on $50 \mathrm{~nm}$ thick $\mathrm{Au}$ film. (d) Scheme of the hybridization of the dimer and the film. The red lever is the hybridized bonding mode of the two particles. The blue lever is still the lever of one particle, but slightly modified by the other one because of the coupling. The pink levers are hybridized states of the particle levers and film mode. between particles (gap $\mathrm{C}$ in Figure 1) in an $\mathrm{R}=30 \mathrm{~nm}$ dimer particle-film system with the thickness of the film varying from $10 \mathrm{~nm}$ to $100 \mathrm{~nm}$. The black dashed curve is the E field from the same gap between two particles on a glass substrate (thickness of $\mathrm{Au}$ film $\mathrm{D}=0 \mathrm{~nm}$ ). The peak at $410 \mathrm{~nm}$ of the dashed curve corresponds to a single un-hybridized particle resonance in the dimer, and the peak at $510 \mathrm{~nm}$ of the dashed curve is the resonant peak of the hybridized bonding mode (we call this the dimer mode, for convenience). The inset in Figure 4(a) show the surface charge distribution of two $\mathrm{R}=30 \mathrm{~nm}$ particles on a glass substrate. Because of the influence of a dielectric substrate, these two modes, indicated by the peak of dashed line, red shift a little bit compared to the pure dimer system. The surface charge distribution on the glass thus helps us to distinguish the single particle mode and the dimer mode in a dimer system. When a layer of gold film is added on the glass substrate, an additional peak appears at longer wavelength because of the strong coupling between the particles and the film. The peak at $510 \mathrm{~nm}$ represents the coupling between single particle mode and film, and the peak at $410 \mathrm{~nm}$ belongs to coupling of high order mode of dimer and film. As shown in Figure 4(d), when the particle dimer is put on a thin film, additional hybridized modes $1^{\prime}$ and $2^{\prime}$, with lower energy, appear because of the hybridization of the bonding mode of the film and the particles (the mode of the film can be distinguished by the charge distribution on the other side, data not shown). When the film becomes thicker, the bonding lever of the film will have larger energy that pushes the hybridized levers to larger energies. The additional peak in Figure 4(a) blue shifts as the thickness of the film increases, and when the thickness is over $40 \mathrm{~nm}$, the shift becomes very small as the coupling between the two sides of the film becomes very weak. Compared to the dashed line in Figure 4(a), which only represents the coupling between particles, this additional peak mainly reflects the coupling between the dimer mode and the film. The resonant peak of the dimer mode $(\sim 510 \mathrm{~nm})$ vanishes slowly as the film becomes thicker; as the effective density of state is larger in thick films make the dimer mode too weak in comparison. There are mainly two peaks emerging from gap between particles and film (gap B, Figure 4(b)). From the charge distributions, we find that the peak at $650-800 \mathrm{~nm}$ is from the coupling between the dimer mode and film, which is at the same position as in Figure 4(a). The peak at about $550 \mathrm{~nm}$ is from the coupling between the resonant mode of a single particle and the film. If the thickness of the film increases, the dimer-film mode blue shifts while the particle-film mode is stable. But from the black curve in Figure 4(b), which is from the gap between a dimer and a $10 \mathrm{~nm}$ thick film, four main peaks are still visible, which are attributed to the hybridization of levers 1 and 2 with the bonding and anti-bonding modes of the film, as shown in Figure 4(d). When the film becomes thicker, the levers $1^{\prime}$ and $2^{\prime}$ will blue shift, and lever $1^{\prime \prime}$ and $2^{\prime \prime}$ red shift because the energy splitting gap between bonding and anti-bonding modes decreases. When the film is thicker than $25 \mathrm{~nm}$, the single particle mode hardly feel the anti-bonding mode of the film, ${ }^{30}$ so levers $1^{\prime \prime}$ and $2^{\prime \prime}$ vanish. By tuning the thickness of the film, the resonant peak of the gap between dimerfilm can be tuned to fit the laser wavelength. For a fixed $50 \mathrm{~nm}$ thickness, we investigated the effect of the dimer's radii. As shown in Figure 4(c), the splitting of the two peaks increases as the radius becomes larger. However, for spheres smaller than $\mathrm{R}=20 \mathrm{~nm}$, the peak from single particle-film coupling slowly disappears because the image dipoles in the film are too close and will start to interact. The high enhancement wavelength range for a freshly made sample can be deduced from the peak shift trend shown in Figures 4(a) and 4(b), combined with Figure 4(c). For the excitation polarization vertical to the axis of the two particles, the coupling between two particles is so weak that the system behaves like two independent single particle-film systems as 
in Figures 1(c) and 3(a). Any other polarization state is a combination of these basic components.

In conclusion, additional resonant modes are generated in a particle-film system by the hybridization between modes of particles and the continuous surface plasmon state of the film. These hybridized modes, which can be tuned by varying the size of particles and the thickness of the film, will lead to huge electric field enhancements in gaps between particles and film. This work gives a clear picture of the plasmon resonance conditions of a particle dimer/film system, with amazing properties that have applications in several research fields, such as, sensing, photocatalysis, and various surface enhanced spectroscopies.

We thank Mikael Svedendahl for discussion and edition of the manuscript and Mikael Käll for helpful discussions. This work was supported by NSFC Grant No. 11204390, Fundamental Research Funds for the Central Universities No.CDJZR 11300003.

${ }^{1}$ H. A. Atwater, Sci. Am. 296, 56 (2007).

${ }^{2}$ V. Giannini, A. I. Fernandez-Dominguez, S. C. Heck, and S. A. Maier, Chem. Rev. 111, 3888 (2011).

${ }^{3}$ E. Ozbay, Science 311, 189 (2006).

${ }^{4}$ S. Zhang, H. Wei, K. Bao, U. Hakanson, N. J. Halas, P. Nordlander, and H. Xu, Phys. Rev. Lett. 107, 096801 (2011).

${ }^{5}$ Z. W. Liu, H. Lee, Y. Xiong, C. Sun, and X. Zhang, Science 315, 1686 (2007).

${ }^{6}$ M. Kall, Nature Mater. 11, 570 (2012).

${ }^{7}$ N. Kakenov, O. Balci, S. Balci, and C. Kocabas, Appl. Phys. Lett. 101, 223114 (2012)

${ }^{8}$ M. Sun and H. Xu, Small 8, 2777 (2012).
${ }^{9}$ H. A. Atwater and A. Polman, Nature Mater. 9, 865 (2010).

${ }^{10}$ H. X. Xu, E. J. Bjerneld, M. Kall, and L. Borjesson, Phys. Rev. Lett. 83, 4357 (1999).

${ }^{11}$ H. J. Lezec, A. Degiron, E. Devaux, R. A. Linke, L. Martin-Moreno, F. J. Garcia-Vidal, and T. W. Ebbesen, Science 297, 820 (2002).

${ }^{12}$ N. Liu and A. Pucci, Nature Mater. 11, 9 (2012).

${ }^{13}$ M. L. Juan, M. Righini, and R. Quidant, Nat. Photonics 5, 349 (2011).

${ }^{14}$ Y. Z. Huang, W. Z. Wang, H. Y. Liang, and H. X. Xu, Cryst. Growth Des. 9, 858 (2009).

${ }^{15}$ H. Y. Liang, Z. P. Li, W. Z. Wang, Y. S. Wu, and H. X. Xu, Adv. Mater. 21, 4614 (2009).

${ }^{16}$ M. T. Sun, Y. Z. Huang, L. X. Xia, X. W. Chen, and H. X. Xu, J. Phys. Chem. C 115, 9629 (2011).

${ }^{17}$ E. Prodan and P. Nordlander, J. Chem. Phys. 120, 5444 (2004).

${ }^{18}$ W. Y. Li, P. H. C. Camargo, X. M. Lu, and Y. N. Xia, Nano Lett. 9, 485 (2009).

${ }^{19}$ W. Y. Li, P. H. C. Camargo, L. Au, Q. Zhang, M. Rycenga, and Y. N. Xia, Angew. Chem., Int. Ed. 49, 164 (2010).

${ }^{20}$ L. M. Tong, V. D. Miljkovic, and M. Kall, Opt. Trapping Opt. Micromanipulation VII 7762, 776200 (2010).

${ }^{21}$ Z. Li, T. Shegai, G. Haran, and H. Xu, ACS Nano 3, 637 (2009).

${ }^{22}$ V. Hafele, F. de Leon-Perez, A. Hohenau, L. Martin-Moreno, H. Plank, J. R. Krenn, and A. Leitner, Appl. Phys. Lett. 101, 201102 (2012).

${ }^{23}$ T. Shegai, Y. Z. Huang, H. X. Xu, and M. Kall, Appl. Phys. Lett. 96, 103114 (2010).

${ }^{24}$ D. Wang, W. Zhu, Y. Chu, and K. B. Crozier, Adv. Mater. 24, 4376 (2012).

${ }^{25}$ J. F. Li, Y. F. Huang, Y. Ding, Z. L. Yang, S. B. Li, X. S. Zhou, F. R. Fan, W. Zhang, Z. Y. Zhou, D. Y. Wu, B. Ren, Z. L. Wang, and Z. Q. Tian, Nature 464, 392 (2010).

${ }^{26}$ E. C. Dreaden, R. D. Near, T. Abdallah, M. H. Talaat, and M. A. El-Sayed, Appl. Phys. Lett. 98, 183115 (2011).

${ }^{27}$ S. Toroghi and P. G. Kik, Appl. Phys. Lett. 100, 183105 (2012).

${ }^{28}$ P. Nordlander and F. Le, Appl. Phys. B: Lasers Opt. 84, 35 (2006).

${ }^{29}$ P. Nordlander and E. Prodan, Nano Lett. 4, 2209 (2004).

${ }^{30}$ F. Le, N. Z. Lwin, J. M. Steele, M. Kall, N. J. Halas, and P. Nordlander, Nano Lett. 5, 2009 (2005). 